\title{
Image edge detection operators based on orthogonal polynomials
}

\begin{abstract}
Orthogonal polynomials (OPs) are beneficial for image processing. OPs are used to reflect an image or a scene to a moment domain, and moments are subsequently used to extract object contours utilised in various applications. In this study, OP-based edge detection operators are introduced to replace traditional convolution-based and block processing methods with direct matrix multiplication. A mathematical model with empirical study results is established to investigate the performance of the proposed detectors compared with that of traditional algorithms, such as Sobel and Canny operators. The proposed operators are then evaluated by using entire images from a well-known data set. Experimental results reveal that the proposed operator achieves a more favourable interpretation, especially for images distorted by motion effects, than traditional methods do.
\end{abstract}

Keyword: Edge detection; Orthogonal polynomials; Gradient image; Sobel operator; Canny operator 